\title{
FACTORES ASOCIADOS CON LA CALIDAD DE VIDA RELACIONADA CON LA SALUD EN PERSONAS CON DIABETES MELLITUS DE LA OBRA SOCIAL UNIVERSITARIA DE CORDOBA
}

\author{
FACTORS ASSOCIATED WITH QUALITY OF LIFE RELATED TO HEALTH IN PERSONS WITH DIABETES \\ MELLITUS OF THE UNIVERSITY SOCIAL WORK OF CORDOBA
}

\author{
Ana C. López ${ }^{1,2,3}$, Eugenio Cechetto ${ }^{1,2}$, Ana N. Aguirre ${ }^{2}$, Marina C. Ontiveros ${ }^{1}$, Claudia V. Roitter ${ }^{1,2}$, \\ Adelaida J. García ${ }^{1}$, Jorgelina Bernet ${ }^{1}$, Jorge A. Aguirre ${ }^{1,2}$
}

\section{Resumen:}

El objetivo del estudio fue describir características clínicas, sociodemográficas y hábitos en adultos con diabetes mellitus y estudiar su asociación con la calidad de vida relacionada con la salud. Se seleccionó una muestra de 311 pacientes del Programa de Diabetes de una obra social universitaria, de la provincia de Córdoba. Se analizaron los datos a como proporciones y los componentes físico y mental del cuestionario SF-36®. El 57,4\% alcanzó un nivel de instrucción terciario o universitario. La hipertensión arterial fue el factor de riesgo cardiovascular más prevalente $(86,8 \%)$, seguida por dislipemia $(50,6 \%)$ y obesidad (46,5\%). El 24,8\% tenía complicación macrovascular y el 29\% microvascular. En el componente físico (CF) de calidad de vida las mujeres tuvieron una chance 4,2 veces mayor de reportar un valor inferior en relación a los varones. Entre los 50 y los 70 años se triplicó y los mayores de 70 años tuvieron 4 veces peor una puntuación en el CF respecto a los más jóvenes. Las complicaciones macro y microvasculares y los que reportaron menos de 2 hábitos de vida saludables tuvieron el doble de chances de puntuar por debajo del promedio general en el CF. La asociación entre complicaciones y peor calidad de vida es un motivo más para alcanzar un buen control metabólico, optimizar el manejo de factores de riesgo, evitando o retrasando la aparición de complicaciones.

Palabras clave: diabetes mellitus; calidad de vida; complicaciones de la diabetes

\section{Abstract:}

The objective of the study was to describe clinical, sociodemographic and habits characteristics in adults with diabetes mellitus and to study their association with health-related quality of life. A sample of 311 patients from the Diabetes Program of a university social work in the province of Córdoba was selected. Data were analyzed for the proportions and physical and mental components of the SF-36® questionnaire. $57.4 \%$ reached a level of tertiary or university education. High blood pressure was the most prevalent cardiovascular risk factor (86.8\%), followed by dyslipidemia (50.6\%) and obesity $(46.5 \%) .24 .8 \%$ had macrovascular complications and $29 \%$ had microvascular complications. In the physical material (FC) of quality of life the women had a 4.2 times greater opportunity to report a lower value in relation to the men. Between the ages of 50 and 70, the number of people in the CF group was three times higher, and those older than 70 years were 4 times worse than the younger ones. Macro and microvascular complications and those reporting less than 2 healthy lifestyles were twice as likely to score below the overall CF average. The association between complications and poorer quality of life is one more reason to achieve a good metabolic control, to optimize the management of risk factors, avoiding or delaying the appearance of complications.

Key words: diabetes mellitus; quality of life; complications of diabetes

\footnotetext{
1 Facultad de Ciencias Médicas, Universidad Nacional de Córdoba

2 DASPU Obra Social Universitaria de Córdoba, Argentina

3 Email de contacto: analopez.af@gmail.com
}

Revista de la Facultad de Ciencias Médicas 2017; 74(4): 


\section{Introducción y objetivos}

La diabetes mellitus es un problema de salud pública que afecta a países desarrollados y en desarrollo ${ }^{1}$. En nuestro país su prevalencia se incrementó significativamente de un $8,4 \%$ en el año 2005 a un $9,8 \%$ en el año 2013. Las proyecciones indican que para 2030 habrá 40 millones de personas con diabetes en América Latina y el Caribe C. $^{2-5}$.

Este trastorno se asocia a un incremento del riesgo de muerte prematura, particularmente porque se vincula con la aparición y el desarrollo de enfermedades cardiovasculares. Las personas con diabetes tienen además un mayor riesgo de padecer ceguera, insuficiencia renal y amputaciones de miembros inferiores.

El estado de salud de las personas con diabetes mellitus se evalúa a partir de indicadores clínicos y estudios complementarios que informan sobre el control metabólico, la presencia de factores de riesgo cardiovascular y de complicaciones relacionadas con esta enfermedad a partir de parámetros definidos, con amplio consenso en la comunidad médico-científica ${ }^{6-10}$. A pesar de múltiples estudios la percepción que tiene el paciente de su estado de salud no es evaluada de manera sistemática en la mayoría de estas recomendaciones. Diversos aspectos de la enfermedad pueden condicionar la calidad de vida de los individuos que la padecen. La cronicidad de la enfermedad, que obliga a realizar controles médicos periódicos, el tratamiento que incluye la adopción de hábitos alimentarios saludables, la realización de actividad física en forma regular, el uso habitual de medicamentos en forma prolongada y la aparición de distintas complicaciones, pueden generar un impacto importante en la calidad de vida de los individuos ${ }^{10}$.

La calidad de vida relacionada con la salud es una medida compuesta por diversas dimensiones que informan sobre el bienestar físico, mental y social de acuerdo a la percepción del paciente. La Organización Mundial de la Salud la define como "la percepción de un individuo de su situación de vida, puesto en el contexto de su cultura y sistema de valores, en relación a sus objetivos, expectativas, estándares y preocupaciones"11,12. Múltiples estudios valoran la calidad de vida relacionada con la salud en pacientes con diabetes mellitus a partir de cuestionarios genéricos y específicos ${ }^{13-15}$. Dos de estos cuestionarios muy utilizados para este fin, el SF-36 ${ }^{\odot}$ y EQ-D5 ${ }^{\odot}$, están validados en nuestro país en la población general ${ }^{16-20}$.

El objetivo de este estudio fue evaluar la calidad de vida relacionada con la salud medida a través del cuestionario $\mathrm{SF}-36^{\circ}$ y relacionarla con las características clínicas, sociodemográficas y hábitos de vida en adultos con diabetes mellitus de la obra social universitaria de la provincia de Córdoba.

\section{Material y métodos}

Se llevó a cabo un estudio descriptivo, observacional, de corte transversal. Se seleccionó una muestra aleatoria simple de 311 pacientes con diabetes mellitus a partir de una población de 1107 pacientes del Programa de Diabetes de una obra social universitaria, de la provincia de Córdoba. Se incluyeron personas de ambos sexos, mayores de 18 años, con diagnóstico de diabetes mellitus tipo 1 o tipo 2 de al menos 12 meses de evolución. Fueron excluidas aquellas personas con deterioro cognitivo, institucionalizadas o con una enfermedad que condicionara una expectativa de vida limitada o una peor calidad de vida relacionada con la salud, diagnóstico de una neoplasia activa, uso de terapia inmunosupresora, o que se negaran a responder y/o participar en el estudio.

Los datos sobre características sociodemográficas y clínicas se obtuvieron de la base de datos del Programa de Diabetes de la obra social, el cuestionario SF- $36^{\odot}$ sobre calidad de vida fue completado por los participantes.

El cuestionario SF-36®, validado en la población argentina, consta de 36 preguntas agrupadas en 8 dimensiones que se relacionan con conceptos de salud y aspectos relacionados con la enfermedad y el tratamiento. Las dimensiones son función física, rol físico, dolor corporal y salud general que conforman el componente físico (CF), y vitalidad, función social, rol emocional y salud mental que conforman el componente mental (CM).

Los factores de riesgo cardiovascular estudiados fueron: hipertensión arterial, dislipemia, obesidad (definida a partir de IMC $\geq 30$ ). Complicaciones microvasculares: retinopatía, nefropatía y neuropatía diabéticas. Complicaciones macrovasculares: cardiopatía isquémica, accidente cerebrovascular y arteriopatía periférica. Variables de laboratorio: Hemoglobina glicosilada (HbA1c, \%), colesterol total (mg/dl).

Los hábitos de vida valorados fueron: Actividad física saludable (AF): Medida a través del Cuestionario Internacional de Actividad Física $\left(\mathrm{IPAQ}^{\odot}\right)$, categorizada en baja, moderada e intensa. Se consideró como saludable a las categorías moderada e intensa del IPAQ ${ }^{\odot}$.

Consumo de frutas y verduras: Se consideró saludable al consumo de 5 o más porciones 
diarias de frutas y/o verduras. Tabaquismo: Se consideraron las categorías no tabaquista o ex tabaquista como saludables. Se construyó una variable "hábitos de vida saludable" a partir de la presencia de 2 o más hábitos de vida saludables (actividad física moderada/intensa, consumo de 5 o más porciones diarias de frutas y/o verduras y estado de no tabaquista o ex-tabaquista).

Las variables categóricas se describieron como proporciones con sus correspondientes intervalos de confianza al 95\% (IC95), las cuantitativas simétricas con la media y el desvío estándar (DE) y las asimétricas con la mediana y el rango. Las comparaciones de medias se realizaron mediante un test $\mathrm{t}$ de Student o $\mathrm{U}$ de Mann-Whitney en caso de no cumplir con los supuestos de homoscedasticidad y distribución normal; las comparaciones de proporciones se realizaron con un test de Chi cuadrado, en ambos casos considerando un error alfa bilateral del $5 \%$.

Los componentes físico y mental del cuestionario SF- $36 \AA$ se consideraron como variable de respuesta en el análisis de regresión logística multivariada. Debido a que son variables de tipo numéricas se tomó a la media de cada componente como punto de corte para generar dos categorías para el análisis de regresión (menor y mayor o igual que la media).

Estimación e interpretación de los coeficientes del modelo: La regresión y sus coeficientes tienen un sentido diferente en comparación con el modelo clásico de regresión, debido a que se realiza una transformación logística a la variable dependiente. Si $\pi$ es la probabilidad de que un individuo bajo estudio pertenezca al grupo de pacientes que "presentan un valor de CF por debajo de la media", la chance de este evento se define como:

$$
\text { chance }=\frac{\pi}{1-\pi}
$$

e indica las veces que ocurre un evento (éxito) por cada no ocurrencia del evento (fracaso). Luego:

$$
\log \left(\frac{\pi}{1-\pi}\right)=\beta_{0}+\beta_{1} X_{1}+\ldots+\beta_{k} X_{k}
$$

Donde $X_{i}$ son las variables regresoras $y$ representan los valores correspondientes a cada individuo en cada una de las variables que intervienen en la ecuación.

Para el análisis del componente físico se retuvieron, por resultar significativas, las variables regresoras: edad, género, hábitos de vida saludables, complicaciones macrovasculares y complicaciones microvasculares. Se ajustó otro modelo para el componente mental, conservando las variables explicativas edad y tiempo de evolución de la enfermedad. Luego, los coeficientes $\mathrm{Bi}$ (desconocidos) de cada modelo logístico se estimaron a partir de la muestra, por el método de máxima verosimilitud mediante el algoritmo de mínimos cuadrados ponderados iterados. Estimados los parámetros fue posible estimar también la probabilidad de éxito para valores particulares de las variables regresoras, en términos de cociente de chances (odds ratio), que indica cuánto mayor (o menor) es la chance de obtener un valor del indicador de calidad de vida que estuviera por encima del valor promedio de la muestra para dicho indicador.

\section{Resultados}

La muestra estuvo constituida por 311 individuos, con un $47.6 \%$ de mujeres (IC95 42.1-53.1). El promedio de edad fue de $46.8( \pm 14.5)$ años para los diabéticos tipo 1 y de $65.6( \pm 10.9)$ años para los diabéticos tipo 2. El 57.4\% (IC95 51.3-62.9) de los encuestados alcanzó un nivel de instrucción terciario o universitario. El 95.2\% de los pacientes (IC95 92.6-97.4) tenía diabetes mellitus tipo 2. El tiempo mediano de evolución de la enfermedad en los diabéticos tipo 2 fue de 9 años con un rango de 47 , y en los diabéticos tipo 1 de 26 años con un rango de 60 .

La hipertensión arterial fue el factor de riesgo cardiovascular más prevalente, con una frecuencia de $86.8 \%$ (IC95 83.2-90.6), seguida por la dislipemia (50.6\%, IC95 44.8-56.8) y la obesidad (46.5\%, IC95 40.6-51.9). El 24.8\% (IC95 20-29.7) presentó al menos una complicación macrovascular y el 29\% (IC95 23.934.5) complicaciones microvasculares. De las macrovasculares la enfermedad coronaria $(20 \%$, IC95 15.2-24.5) fue la de mayor frecuencia y de las microvasculares la nefropatía diabética (18.7\%, IC95 14.2-22.9). El promedio de hemoglobina glicosilada (HbA1c) fue de $7.2 \%$ $( \pm 1.44)$ y el de colesterol de $183 \mathrm{mg} / \mathrm{dl}( \pm 40.6)$. La mayoría de los pacientes tenía tratamiento con antidiabéticos orales $(69.1 \%$, IC95 63.974.2), un 13.7\% (IC95 9.8-17.5\%) usaba la combinación de antidiabéticos orales e insulina y un $10.4 \%$ (IC95 7-13.8\%) insulinoterapia exclusivamente. Un 6.8\% (IC95 4-9.6\%) no recibía tratamiento farmacológico. En el grupo con complicaciones microvasculares la media de $\mathrm{HbA1c}$ fue significativamente más elevada (7.54 vs 7.06, $p=0.014$ ), esta asociación no fue estadísticamente significativa en el caso de las complicaciones macrovasculares ( 7.29 vs 7.17 , , $\mathrm{p}=0.4$ ). En las personas con complicaciones macrovasculares la prevalencia de hipertensión 
arterial o dislipemia fue mayor que en los que no presentaban este antecedente $(98.7$ versus $82.8 \%$ para la hipertensión arterial, $\mathrm{p}<0.0001$ y 70.1 versus $44.2 \%$ para la dislipemia, $\mathrm{p}<0.0001$ ). Las complicaciones microvasculares fueron más frecuentes en los pacientes que recibían insulina (OR 2.58, IC 95\% 1.81-3.46, p<0.0001); no se observaron diferencias en relación a la insulinoterapia y las complicaciones macrovasculares.

En relación a los hábitos de vida, el 39.9\% (IC95 34.3-45.4) refirió realizar actividad física considerada saludable según IPAQ ${ }^{\odot}$. El $68.3 \%$ (IC95 63-73.3) manifestó consumir frutas 5 o más días por semana y el 75.3\% (IC95 70.3-80.3) refirió consumir verduras 5 o más días por semana, en tanto que el 20.5\% (IC95 15.7-24.9) reportó comer 5 o más porciones de frutas y/o verduras diarias. El 19.3\% (IC95 15-23.5) se identificó como tabaquista actual. Considerando hábitos saludables a la actividad física, el consumo de frutas y verduras y no fumar, el 49.7\% (IC95 44.1-54.9) manifestó cumplir con un hábito saludable, $30.9 \%$ (IC95 26-36.5) dos hábitos y el 9.9\% (IC95 6.6-12.8) con los 3 hábitos considerados saludables; un porcentaje similar, 9.5\% (IC95 6.3-13.2), no cumple con ningún hábito saludable. En las Tabla 1 y 2 se aportan más detalles sobre las características de los pacientes.

\begin{tabular}{|c|c|}
\hline \multicolumn{2}{|c|}{$\begin{array}{l}\text { TABLA 1.- Características clínicas y sociodemográficas de los } \\
\text { pacientes de la muestra. }\end{array}$} \\
\hline $\begin{array}{l}\text { Características clínicas y sociodemográficas de } \\
\text { la muestra }\end{array}$ & $\mathrm{N}(\%)$ \\
\hline Género (mujeres) & $148(47.6)$ \\
\hline Edad en años (media \pm DE) & $64.7( \pm 11.8)$ \\
\hline Diabéticos tipo I & $46.8( \pm 14.5)$ \\
\hline Diabéticos tipo II & $65.6( \pm 10.9)$ \\
\hline \multicolumn{2}{|l|}{ Nivel de instrucción } \\
\hline Sin instrucción formal & $1(0.3)$ \\
\hline Primario & $45(14.5)$ \\
\hline Secundario & $86(27.7)$ \\
\hline Terciario/Universitario & $178(57.4)$ \\
\hline \multicolumn{2}{|l|}{ Tipo de diabetes } \\
\hline DM tipo 1 & $15(4.8)$ \\
\hline DM tipo 2 & $296(95.2)$ \\
\hline \multicolumn{2}{|l|}{ Tiempo de evolución en años (mediana; rango) } \\
\hline DM tipo 1 & $26(60)$ \\
\hline DM tipo 2 & $9(47)$ \\
\hline \multicolumn{2}{|l|}{ Tratamiento recibido } \\
\hline Tratamiento no farmacológico & $21(6.8)$ \\
\hline Insulina & $32(10.4)$ \\
\hline Antidiabéticos orales e insulina & $42(13.7)$ \\
\hline Antidiabéticos orales & $212(69.1)$ \\
\hline
\end{tabular}

\begin{tabular}{|c|c|}
\hline Hipertensión arterial & $269(86.8)$ \\
\hline Dislipemia & $157(50.6)$ \\
\hline \multicolumn{2}{|l|}{ Hábito tabáquico } \\
\hline Tabaquista actual & $59(19.3)$ \\
\hline Ex tabaquista & $68(22.2)$ \\
\hline No tabaquista & $179(58.5)$ \\
\hline \multicolumn{2}{|l|}{ Índice de masa corporal (IMC) } \\
\hline Delgadez $(\leq 19.9)$ & $3(1)$ \\
\hline Normal (20-24.9) & $48(15.5)$ \\
\hline Sobrepeso (25-29.9) & $115(37.1)$ \\
\hline Obesidad $(\geq 30)$ & $144(46.5)$ \\
\hline $\mathrm{HbA} 1 \mathrm{c}<7.5 \%$ & $214(69.9)$ \\
\hline Colesterol total > $200 \mathrm{mg} / \mathrm{dl}$ & $97(31.3)$ \\
\hline
\end{tabular}

\begin{tabular}{|c|c|}
\hline \multicolumn{2}{|c|}{ TABLA 2.- Presentación de complicaciones y hábitos de vida. } \\
\hline Tipo de complicaciones y hábitos & $\mathrm{N}(\%)$ \\
\hline \multicolumn{2}{|l|}{ Complicaciones } \\
\hline Sin complicaciones & $178(57.2)$ \\
\hline Complicaciones microvasculares & $90(28.9)$ \\
\hline Nefropatía & $58(18.7)$ \\
\hline Neuropatía & $37(11.9)$ \\
\hline Retinopatía & $23(7.4)$ \\
\hline Complicaciones macrovasculares & $77(24.8)$ \\
\hline Cardiopatía isquémica & $62(19.9)$ \\
\hline Accidente cerebrovascular & $18(5.8)$ \\
\hline Arteriopatía periférica & $15(4.8)$ \\
\hline Ambas & $35(11.2)$ \\
\hline \multicolumn{2}{|l|}{ Hábitos saludables } \\
\hline $\begin{array}{l}\text { Consumo de frutas } 5 \text { a } 7 \text { días por } \\
\text { semana }\end{array}$ & 227 (74.9) \\
\hline $\begin{array}{l}\text { Consumo de verduras } 5 \text { a } 7 \text { días por } \\
\text { semana }\end{array}$ & 205 (68.1) \\
\hline $\begin{array}{l}\text { Consumo de } 5 \text { porciones de frutas y/o } \\
\text { verduras al día }\end{array}$ & $60(20.5)$ \\
\hline Actividad física saludable & $122(39.9)$ \\
\hline \multicolumn{2}{|l|}{ Cantidad de hábitos de vida saludables } \\
\hline Ninguno & $29(9.5)$ \\
\hline Uno & $151(49.7)$ \\
\hline Dos & $94(30.9)$ \\
\hline Tres & $30(9.9)$ \\
\hline
\end{tabular}

Los promedios de los indicadores de calidad de vida fueron $47.52( \pm 8.77)$ y $47.84( \pm 9.65)$ para los componentes físico y mental, respectivamente. En la Figura 1 se describen los promedios de las dimensiones de los componentes físico y mental en el grupo total y en relación al género. 


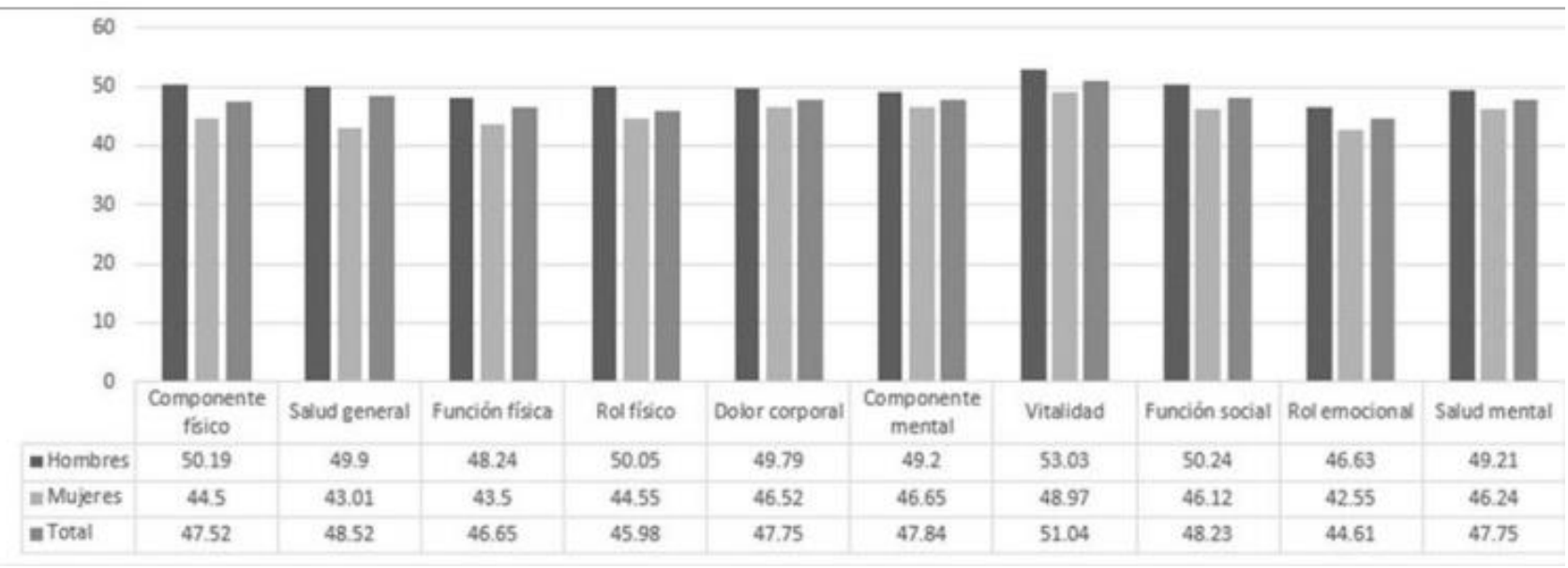

Figura 1. Promedios de las dimensiones de los componentes físico y mental en el grupo total y en relación al género.

En el análisis de regresión logística se ajustó un modelo para el componente físico en el que se encontró asociación entre valores inferiores a la media en este indicador y la edad, género, hábitos de vida y complicaciones; no se pudo ajustar un modelo adecuado entre las variables regresoras y el indicador del componente mental. En la Tabla 3 se muestran las variables que resultaron significativas para el modelo obtenido, las estimaciones de los coeficientes $\beta \mathrm{i}$, el estadístico de Wald y su significación estadística, y los cocientes de chances (odds ratio) correspondientes.

\begin{tabular}{|c|c|c|c|c|c|}
\hline \multicolumn{6}{|c|}{$\begin{array}{l}\text { TABLA 3.- Modelo de regresión logística multivariado para el } \\
\text { indicador componente físicointas variables regresoras. }\end{array}$} \\
\hline & & $\beta \mathrm{i}$ & Wald & OR** & $\mathrm{p}$ \\
\hline \multicolumn{2}{|l|}{ Constante } & $\overline{-}$ & 31,1 & 0,04 & $<0.0001$ \\
\hline \multirow{2}{*}{ Género } & Masculino* & - & - & 1 & - \\
\hline & Femenino & 1,43 & 26,67 & 4,17 & $<0.0001$ \\
\hline \multirow{3}{*}{ Edad } & $<50$ años* & - & - & 1 & - \\
\hline & 50-70 años & 1,15 & 4,96 & 3,15 & 0,026 \\
\hline & $>70$ años & 1,5 & 7,64 & 4,46 & 0,006 \\
\hline \multirow{2}{*}{$\begin{array}{l}\text { Complicaciones } \\
\text { macrovasculares }\end{array}$} & No* & - & - & 1 & - \\
\hline & $\mathrm{Si}$ & 0,68 & 3,46 & 1,98 & 0,035 \\
\hline \multirow{2}{*}{$\begin{array}{l}\text { Complicaciones } \\
\text { microvasculares }\end{array}$} & No* & - & - & 1 & - \\
\hline & $\mathrm{Si}$ & 0,92 & 9,81 & 2,56 & 0,002 \\
\hline \multirow{2}{*}{$\begin{array}{l}\text { Hábitos de vida } \\
\text { saludables }\end{array}$} & 2 o más* & - & - & 1 & - \\
\hline & Menos de 2 & 0,82 & 9 & 2,28 & 0,03 \\
\hline
\end{tabular}

En el cuestionario de calidad de vida las mujeres tuvieron una chance 4.2 veces mayor de reportar un valor inferior al promedio general del componente físico en relación a los varones. La edad también demostró ser un factor importante que influyó sobre la chance de que un individuo presente valores menores del indicador del componente físico. Sobre este punto se observó que las personas con edades comprendidas entre los 50 y los 70 años tuvieron 3 veces más chances de tener una puntuación en el CF inferior al promedio general en relación a personas más jóvenes, mientras que en los mayores de 70 años se cuadruplicó en relación al grupo de menores a 50 años.

Los individuos que sufrieron complicaciones macro y microvasculares y los que reportaron menos de 2 hábitos de vida saludables tuvieron cerca del doble de chances de puntuar por debajo del promedio general en el componente físico (Tabla 3).

\section{Discusión}

La diabetes mellitus es una enfermedad de gran importancia sanitaria. Múltiples estudios han descripto sus características desde el punto de vista sociodemográfico y clínico, otros estudios miden el impacto mediante la carga de enfermedad. Al considerar la calidad de vida se valora la percepción del individuo en relación a su estado de salud, este abordaje pone en escena a la persona y su percepción desde diferentes dimensiones, una mirada que complementa los estudios epidemiológicos y considera el análisis de los determinantes de la calidad de vida en el desafío de realizar un abordaje integral de la salud de las personas. La mayoría de los estudios sobre el tema son transversales, insuficientes para identificar causalidad, pero muestran una asociación entre ciertas características de los pacientes y la calidad de vida. De esta forma, la información obtenida puede ser de gran utilidad en el seguimiento individual y la generación de políticas sanitarias. Se han publicado pocos estudios en nuestro país sobre calidad de vida valorada a partir del cuestionario SF-36 ${ }^{\odot}$, por lo cual consideramos relevante el aporte de este estudio a la epidemiología local. 
En múltiples investigaciones se ha demostrado que la diabetes mellitus tiene un impacto negativo en la calidad de vida relacionada con la salud $^{16,21-31}$. En esta investigación se identificaron factores que se asocian a una peor calidad de vida en personas con diabetes mellitus en un modelo multivariado.

Se pudo ajustar un modelo de regresión logística multivariado que mostró una asociación entre distintas características de los pacientes y una peor puntuación en las dimensiones agrupadas en el componente físico. A diferencia de otros estudios, no se pudo ajustar un modelo adecuado en relación con el componente mental. Sin embargo, en el análisis bivariado, se encontró una correlación positiva, débil pero estadísticamente significativa, entre la edad y el puntaje de la calidad de vida en el CM (Rho $0.143, p=0.013$ ), similar a la hallada en otras publicaciones $^{27,28}$.

La edad fue el factor de mayor influencia en el puntaje del CF, el deterioro se incrementó por encima de los 50 años, y en mayor medida por encima de los 70 años, fenómeno que se observa también en otros estudios de calidad de vida tanto en personas con diabetes como en personas sanas o con otros problemas de salud ${ }^{8,16,22,29-31}$. Llamó la atención una tendencia no significativa, en el análisis bivariado, hacia un mayor puntaje en el $\mathrm{CM}$ en pacientes de mayor edad, una relación que ya fue referida en otros estudios, sin embargo no se pudeo ajustar un modelo de regresión logística adecuado en este indicador.

En relación al género nuestros datos coinciden con otros publicados, en los que se evidencia una peor puntuación en el CF de la calidad de vida en las mujeres ${ }^{16,23,25,28-31}$. No encontramos asociación entre este indicador y el nivel educativo, probablemente debido a que al estudiar afiliados a una obra social universitaria hubo una elevada proporción de sujetos con un nivel de instrucción terciario o universitario ${ }^{29} \mathrm{~A}$ diferencia de otras publicaciones no encontramos, ni en el análisis bivariado ni en el modelo multivariado, una asociación entre la calidad de vida, los factores de riesgo cardiovascular más frecuentes (hipertensión arterial y dislipemia) y variables de laboratorio (hemoglobina glicosilada y colesterol total) ${ }^{27,28}$. El carácter asintomático de estos indicadores podría explicar el hecho de que no generen por sí mismos un impacto en la calidad de vida sino a través de sus complicaciones, ya que en el análisis bivariado, se identificó una asociación estadísticamente significativa entre las cifras de hemoglobina glicosilada y colesterol total y la presencia de complicaciones micro y macrovasculares, respectivamente, así como entre la presencia de hipertensión arterial y dislipemia y las complicaciones macrovasculares. Por tanto, creemos que estas características se comportarían como subrogadas y que el impacto en la calidad de vida se produciría por la presencia de complicaciones de la enfermedad, como lo demuestra la menor puntuación en el CF de la calidad de vida en los pacientes con complicaciones, ajustando por otras variables incluidas en el modelo. Esta asociación entre calidad de vida y complicaciones en pacientes con diabetes mellitus fue descripta consistentemente en estudios previos 21,22,24,25,29- 31. En algunos estudios se demostró que la obesidad, por si misma, puede condicionar una peor calidad de vida tanto en la dimensión física como en la mental y en personas con y sin diabetes mellitus, pero no pudimos demostrar esta asociación en nuestro estudio ${ }^{31}$.

Otras publicaciones han descripto la relación entre la utilización de insulina y una peor calidad de vida, habiéndose hipotetizado que el uso de insulina estaría asociado con mayor frecuencia a un diagnóstico de diabetes mellitus tipo 10 a fases más avanzadas de la enfermedad y que estos hechos podrían condicionar una peor calidad de vida ${ }^{28,30,31}$. En nuestro estudio un $10 \%$ de la muestra estudiada recibía tratamiento con insulina. No encontramos en este grupo una peor calidad de vida en relación a las personas en tratamiento con antidiabéticos orales, antidiabéticos orales e insulina o tratamiento no farmacológico. A pesar de ello, el subgrupo de pacientes bajo insulinoterapia exclusiva presentó una mayor probabilidad de complicaciones microvasculares. El tratamiento con insulina podría tener, por tanto, una relación con la calidad de vida similar a la descripta, es decir, de manera subrogada a traves de su asociación con las complicaciones, en este caso microvasculares.

De manera similar a otros estudios, encontramos que la presencia dos o mas hábitos de vida saludables se asocia a una mejor calidad de vida en el $\mathrm{CF}^{26,29}$

Creemos que la asociación entre la presencia de complicaciones y una peor calidad de vida es un motivo más para perseguir el cumplimiento de los objetivos, alcanzar un buen control metabólico y optimizar el manejo de los factores de riesgo, evitando o retrasando de esta manera la aparición de complicaciones a largo plazo.

Este estudio ofrece pruebas para fortalecer, en el abordaje de los pacientes con diabetes mellitus, las recomendaciones en relación a hábitos de vida saludable dada la asociación encontrada entre éstos y una mejor calidad de vida. 
La principal limitación de esta investigación se debe a que la muestra estudiada proviene de una obra social universitaria en la que los pacientes se encuentran en un programa de enfermedades crónicas, muestra que podría no representar a la población general de diabéticos; a pesar de ello, encontramos resultados similares en estudios realizados en otras poblaciones. Por otro lado, la falta de un grupo de control no permite estudiar el efecto propio de la diabetes mellitus sobre la calidad de vida de los pacientes la obra social.

\section{Agradecimientos}

La investigación fue realizada con un subsidio de la Secretaria de Ciencia y Técnica de la Universidad Nacional de Córdoba. Agradecemos Ines Ahumada del Instituto de Estadística y Demografía de la Facultad de Ciencias Económicas, Universidad Nacional de Córdoba.

\section{Bibliografía}

1. World Health Organization. World Health Statistics 2014. En: http://apps.who.int/iris/bitstream/10665/112738/1/9789240692671 eng.p df; consultado el 01/12/2015.

2. Programa nacional de prevención y control de la Diabetes mellitus (PRONADIA). 1999. Ministerio de Salud y Acción Social de la República Argentina.

http://www.msal.gov.ar/images/stories/bes/graficos/0000000390cnt-201402 resolucion-301-99-aprobacion-pronadia.pdf; consultado el $01 / 02 / 2016$.

3. La diabetes muestra una tendencia ascendente en las américas. En http://www.paho.org/chi/index.php?option=com content\&view=article\&id= 467:la-diabetes-muestra-tendencia-ascendente-americas; consultado el 21/02/2016.

4. Tercer Encuesta Nacional de Factores de Riesgo. 2013. Ministerio de Salud de la Nación. República Argentina. En: http://www.msal.gob.ar/images/stories/publicaciones/pdf/11.09.2014tercer-encuentro-nacional-factores-riesgo.pdf; consultado el 20/02/2016.

5. Ferrante $D$, Linetzky $B$, Konfino J, et al. Encuesta Nacional de Factores de Riesgo 2009: Evolución de la epidemia de enfermedades crónicas no transmisibles en Argentina. Estudio de corte transversal. Rev Argent Salud Pública 2011; 2: 34-42.

6. Executive Summary: Standards of Medical Care in Diabetes - 2010. Diabetes Care 2010; 33:S4-S10.

7. Type 2 diabetes: National clinical guideline for management in primary and secondary care (update). National Collaborating Centre for Chronic Conditions. London. Royal College of Physicians, 2008.

8. Management of diabetes: A national clinical guideline. Scottish Intercollegiate Guidelines Network. 2010.

9. Guía de Práctica Clínica Nacional sobre Prevención, Diagnóstico y Tratamiento de la Diabetes Mellitus Tipo 2 para el primer nivel de atención. Ministerio de Salud de la Nación. República Argentina. 2008.

10. Grupo de Trabajo de la Guía de Práctica Clínica sobre Diabetes tipo 2. Guía de Práctica Clínica sobre Diabetes tipo 2. Madrid: Plan Nacional para el SNS del MSC. Agencia de Evaluación de Tecnologías Sanitarias del País Vasco; 2008. Guías de Práctica Clínica en el SNS: OSTEBA № 2006/08.

11. Osorio Parraguez P, Torrejón MJ, Anigstein MS. Calidad de vida en personas mayores en Chile. Revista Mad 2011; (24): 61-75.

12. Schwartzmann L. Calidad de vida relacionada con la salud: Aspectos conceptuales. Ciencia y Enfermeria 2003; 9:9-21.

13. Aguiar C, Fernandes A, Ferrer Carvalho, et al. Instrumentos de Avaliação de Qualidade de Vida Relacionada à Saúde no Diabetes Melito. Arq Bras Endocrinol Metab 2008; 52:931-9.

14. Millan M. Cuestionario de calidad de vida específico para la diabetes mellitus (EsDQOL) Aten Primaria 2002. 15 de mayo. 29 (8): 517-521.

15. López-Carmona JM, Rodríguez Moctezuma R. Adaptación y validación del instrumento de calidad de vida Diabetes 39 en pacientes Mexicanos con Diabetes Mellitus tipo 2. Salud Pública de México 2006; 48:200-11.

16. Mata Cases M, Roset Gamisans M, Badia Llach X et al. Impacto de la diabetes mellitus tipo 2 en la calidad de vida de los pacientes tratados en las consultas de atención primaria en España. Aten Primaria 2003; 31:493-9.

17. Herdman M, Badia X, Berra S. El EuroQol-5D: una alternativa sencilla para la medición de la calidad de vida relacionada con la salud en atención primaria. Aten Primaria 2001. 15 de octubre. 28 (6): 425-9.
18. Hervás A, Zabaleta A, De Miguel G, et al. Calidad de vida relacionada con la salud en pacientes con diabetes mellitus tipo 2. An Sist Sanit Navar 2007; 30: 45-52.

19. Augustovsky FA, Lewin G, Elorrio EG, Rubinstein A. The ArgentineSpanish SF-36 Health Survey was successfully validated for local outcome research. J Clin Epidemiol 2008; 61: 1279-84.

20. Augustovski $F$, Irazola $V$, Velazquez $A$, et al. Argentine Valuation of the EQ-5D Health States International Society for Pharmacoeconomics and Outcomes Research. ISPOR 2008; 12: 587-96.

21. Clark P, Simon J, Cull C, et al. Assessing the Impact of Visual Acuity on Quality of Life in Individuals With Type 2 Diabetes Using the Short Form36. Diabetes Care 2006; 29:1506-11.

22. De Berardis G, Pellegrini F, Franciosi M, et al. Longitudinal Assessment of Quality of Life in Patients With Type 2 Diabetes and Self-Reported Erectile Dysfunction. Diabetes Care 2005; 28:2637-43.

23. Goldney R, Phillips $P$, Fisher L, et al. Diabetes, Depression, and Quality of Life. Diabetes Care 2004: 27:1066-70.

24. U.K. Prospective Diabetes Study Group: Quality of life in type 2 diabetic patients is affected by complications but not by intensive policies to improve blood glucose or blood pressure control (UKPDS 37). Diabetes Care 1999; 22:1125-36.

25. Coffey JT, Brandle $M$, Zhou $\mathrm{H}$, et al. Valuing health related quality of life in diabetes. Diabetes Care 2002; 25:2238-43.

26. Li Ch, Ford E, Mokdad A, et al. Clustering of Multiple Healthy Lifestyle Habits and Health-Related Quality of Life Among U.S. Adults With Diabetes. Diabetes Care 2007; 30:1770-6.

27. Mena Martin FJ, Martin Escudero JC, Simal Blanco F, et al. Diabetes mellitus tipo 2 y calidad de vida relacionada con la salud: Resultados del Estudio Hortega. An Med Interna (Madrid) 2006; 23: 357-60.

28. Thommasen HV, Zhang W. Health-related quality of life and type 2 diabetes: A study of people living in the Bella Coola Valley. BC Medical Journal 2006; 48: 272-8.

29. Glasgow RE, Ruggiero L, Eakin EG, et al. Quality of life and associated characteristics in a large national sample of adults with diabetes. Diabetes Care 1997; 20: 562-7.

30. Rubin RR, Peyrot M. Quality of life and diabetes. Diabetes Metab Res Rev 1999; 15: 205-18.

31. Wändel PE. Quality of life of patients with diabetes mellitus. An overview of research in primary health care in the Nordic countries. Scand J Prim Health Care 2005; 23: 68-74. 